\title{
Estimation of the Wind Component Normal to a Radar Beam
}

\author{
Peter T. MaY*, Susumu Kato, Shoichiro FukaO, Toshitaka Tsuda, and Toru Sato \\ Radio Atmospheric Science Center, Kyoto University, Uji, Kyoto, Japan
}

(Received January 18, 1988; Revised June 15, 1988)

\begin{abstract}
A method is presented whereby a crude estimate of the total wind speed with a single Doppler radar observation may be obtained by making use of the spectral width of the returned signal. This method is demonstrated using data from the MU radar of Japan. However, the accuracy of the estimate is severely limited by spectral broadening due to windshear and turbulence within the radar pulse volume. Therefore the practical applications of this method are limited.
\end{abstract}

\section{Introduction}

The purpose of this paper is to show that under some conditions it is possible to estimate the magnitude of the velocity of the wind perpendicular to a radar beam direction with just a single observation. This estimate uses information of the observed spectral width as well as the line-of-sight Doppler shift. However, this is only possible under the very strict conditions that the spectral width due to the finite beamwidth (beam-broadening) is much greater than contributions to the spectral width from such processes as windshear within the pulse volume and turbulence. This condition is often true for wind profilers (e.g., HoCKING, 1986) but is rarely so for Doppler weather radars (DovIAK and ZRNIC, 1984). The accuracy of this estimate, under the above conditions, is discussed and radar data from the $\mathrm{MU}$ radar of Japan is used to verify that it is possible to make these estimates.

\section{Estimation of the Wind Component}

We assume that the shape of the observed power spectrum of a radar signal is well approximated by a Gaussian profile with a white noise component of the spectrum, which is subtracted, i.e., $S(u)=P \exp \left[-\left(u-u_{\mathrm{d}}\right)^{2} / 2 \sigma_{\mathrm{u}}^{2}\right]$ where the frequency is in units of $\mathrm{ms}^{-1}$. The observed spectral width of a radar signal is $\sigma_{\mathrm{u}}$ and $\sigma_{\mathrm{u}}^{2}$ may be considered as the sum of several contributions, i.e.,

$$
\sigma_{\mathrm{u}}^{2}=\sigma_{\mathrm{b}}^{2}+\sigma_{\mathrm{s}}^{2}+\sigma_{\mathrm{t}}^{2}+\sigma_{\mathrm{w}}^{2}
$$

where $\sigma_{\mathrm{b}}$ is the spectral broadening due to the finite beamwidth, $\sigma_{\mathrm{s}}$ is the spectral

\footnotetext{
*Currently NRC/NOAA Resident Research Associate, Wave Propagation Laboratory, NOAA, Boulder, CO, U.S.A.
} 
width due to windshear within the pulse volume, $\sigma_{\mathrm{t}}$ is the contribution from turbulence and $\sigma_{\mathrm{w}}$ is the broadening due to the data window function. Here we assume that the second and third terms on the righthand side are small compared to the first (retaining the fourth as this is a simple numerical correction which may be significant for small spectral widths). The beambroadening arises from the finite beamwidth since the backscatter is coming from a range of angles, and therefore the signal has contributions from a number of projections of the wind velocity. For a circularly symmetric radar beam we can write the beam-broadening term, to a good approximation, as the product of the magnitude of the horizontal wind, $V$, with the beamwidth $\Delta \theta$ (defined as the $6 \mathrm{~dB}$ two-way beamwidth in radians) and a numerical factor $k$ (ATLAS, 1964; HoCKING, 1985).

$$
\sigma_{\mathrm{b}}^{2} \approx k \cdot V^{2} \cdot \Delta \theta^{2}=k\left(u^{2} \cdot \Delta \theta^{2}+v^{2} \cdot \Delta \theta^{2}\right),
$$

where $u$ and $v$ are the components of the horizontal wind in the plane and perpendicular to the plane of the radar beam respectively. The vertical component may contribute with a term $(w \cdot \theta \cdot \Delta \theta)$ where $\theta$ is the mean angle from the zenith of the radar beam, but this is neglected because $w$ is usually much less than $V$ and $\theta$ is of the order of 0.2 radians. For these definitions of spectral width and beamwidth $k$ has the value of $1 /(8 \ln 4)$. From Eqs. (1) and (2), neglecting the shear and turbulence terms in (1), and rearranging we obtain an estimate for the wind component transverse to the radar beam as:

$$
v^{2} \approx \sigma_{\mathrm{u}}^{2} /\left[k \cdot \Delta \theta^{2}\right]-u^{2}-\sigma_{\mathrm{w}}^{2} /\left[k \cdot \Delta \theta^{2}\right] .
$$

Note that our estimate of $u$ is obtained from the line-of-sight Doppler shift $u_{\mathrm{r}}$ which is equal to $u \cdot \sin \theta$. The window broadening is estimated by the 2 nd central moment of the mainlobe of the Fourier transform of the (rectangular) window function. The first few sidelobes may also contribute as for realistic spectra these lie within the "true" spectrum, but these are suppressed in power by around $13 \mathrm{~dB}$ and so are ignored. Contributions from the far sidelobes will add to the apparent noise level of the spectrum. For the radar parameters described in Table 1 a value for $\sigma_{\mathrm{w}}$ of 0.115 $\mathrm{ms}^{-1}$ is obtained, which is much smaller than the observed spectral widths.

Assuming that the errors in the determination of the mean Doppler shift and the spectral width are independent we can express the variance of the estimate in $v^{2}$ as:

$$
\operatorname{var}\left(v^{2}\right)=\left(4 \sigma_{\mathrm{u}}^{2} /\left[k^{2} \cdot \Delta \theta^{4}\right]\right) \operatorname{var}\left(\sigma_{\mathrm{u}}\right)+4 u^{2} \operatorname{var}(u)
$$

The above equation for the variance of our estimate of $v$ is strongly dependent on the precision of our estimate of $\sigma_{\mathrm{u}}$ since the value of $\Delta \theta$ is usually small. However, it should be stressed that the above estimate may be strongly biased to give too large an estimate in the presence of turbulence and windshear. For radar soundings performed with the MU radar, which uses similar operating parameters to many 
Table 1. Operating parameters of the MU radar for this experiment.

\begin{tabular}{ll}
\hline Frequency & $46.5 \mathrm{M} \mathrm{Hz}$ \\
Peak transmitter power (1 $\mu \mathrm{sec}$ pulse) & $1 \mathrm{MW}$ \\
Range resolution & $150 \mathrm{~m}$ \\
Ranges sampled & $1-19.2 \mathrm{~km}$ \\
Pulse repetition period & $400 \mu \mathrm{sec}$ \\
Post-coherent averaging time & $0.07 \mathrm{sec}$ \\
Number of data points for power spectrum & 128 \\
Number of spectra averaged for moment estimates & 5 \\
Beam directions and angle from the zenith & North $10^{\circ}$ \\
& East $10^{\circ}$ \\
& South $10^{\circ}$ \\
Beamwidth (6 dB two way or $3 \mathrm{~dB} 1$ way) & West $10^{\circ}$ \\
\hline
\end{tabular}

MST radars (Table 1), the expected standard deviation of $v^{2}$ from the above equation is approximately $500 \mathrm{~m}^{2} \mathrm{~s}^{-2}$ using the results of YАМАМОТО et al. (1988) for estimates of the uncertainty in $u$ and $\sigma_{\mathrm{u}}$, with a typical spectral width of $0.7 \mathrm{~ms}^{-1}$.

\section{Experimental Results}

The MU radar of Japan has been used to test the above conjectures. This radar has been extensively described elsewhere (FUKAO et al., 1985a, b) and will not be described in detail here. The radar parameters are given in Table 1, but note that only the northward and the eastward pointing beams $10^{\circ}$ from the zenith were used in this analysis. The heights which are analyzed here are between 1.5 and $5 \mathrm{~km}$, where the windshear is small and the signal-to-noise ratio is very high $(\geq 15 \mathrm{~dB})$, although there is data above and below this region. This range is only a limit applied by signal-to-noise considerations and it may be that this method should work better in the lower statosphere where turbulence is usually small.

Data were taken during a 2 hour period on January 23, 1987, a few hours preceeding the passage of a cold front. It is noticable that the wind field in the lower to middle troposphere had little shear over the depth of the region analyzed (Fig. 1) during this period. Estimates of the northward component of the wind using the observed spectral width from the beam pointed towards the east have been compared with the estimates of the wind using the northward beam, and similarly the magnitude of the eastward component inferred from the spectral width of the northward beam is compared to the observed values of the eastward wind component (Fig. 2). The group with the small velocity is the northward component of the wind and the larger velocity group is the eastward component. Estimates of the wind using single soundings $(\sim 1 \mathrm{~min})$ are shown in Fig. 2(a) while Figs. 2(b) and 2(c) show the results when the estimated line-of-sight winds and spectral widths are averaged over 9 and $100(\sim 9$ and $100 \mathrm{~min})$ soundings respectively The values of $v^{2}$ 


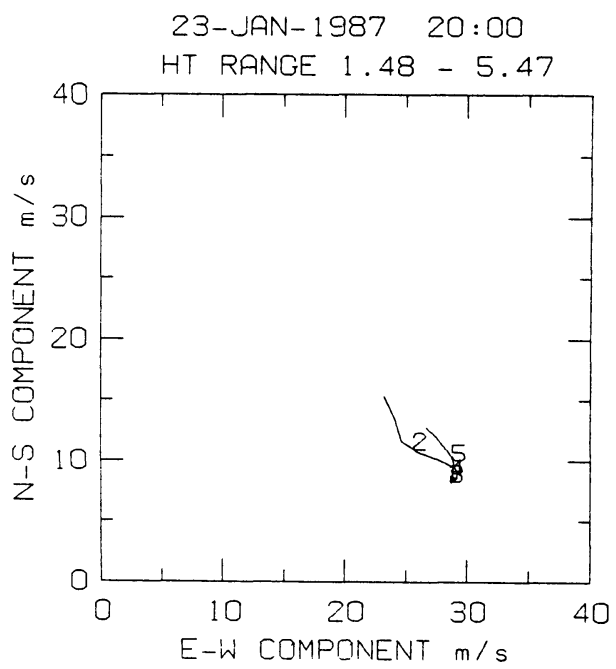

Fig. 1. Hodograph of the horizontal wind estimates averaged over the period of the analysis. These estimates used the north and east beams only. The numbers on the hodograph give the altitude in $\mathrm{km}$.

and $|v|$ are shown, but note that when the estimate of $v^{2}$ is negative the imaginary $|v|$ are not plotted.

It is clear from these observations that the spread in the determination of $v^{2}$ is similar for the two beam directions, as expected, but this means that the accuracy of the determination of the magnitude of the transverse velocity, $|v|$, is larger for the case where the wind normal to the radar beam is larger than the component along the radar beam direction. The observed scatter of $v^{2}$ (north or east beams) has contributions from time and height variations of the wind as well as large random errors so that the observed scatter of around $700 \mathrm{~m}^{2} \mathrm{~s}^{-2}$ is reasonably consistent with theoretical expectations. A clear bias towards overestimating the wind speed is seen in these results. This is consistent with the observed spectrum being broadened from that due just to the beam broadening and window effects. The above analysis has ignored the spectral broadening due to windshear and turbulence. The magnitude of the bias is consistent with an additional term with a spectral width of only 0.4 $\mathrm{ms}^{-1}$ and which is most likely due to clear air turbulence as the observed windshear is small and the radar will only see a small projection of the shear. HocKING (1986) found using the SOUSY VHF radar that turbulence in the troposphere and stratosphere was too weak to be observed, but given the pre-frontal conditions and the observation of some convection in the vertically pointing radar beam, it is probable that turbulence was also contributing to the spectral width. Nevertheless it is interesting that even this level of agreement could be obtained with such a simple estimate. 

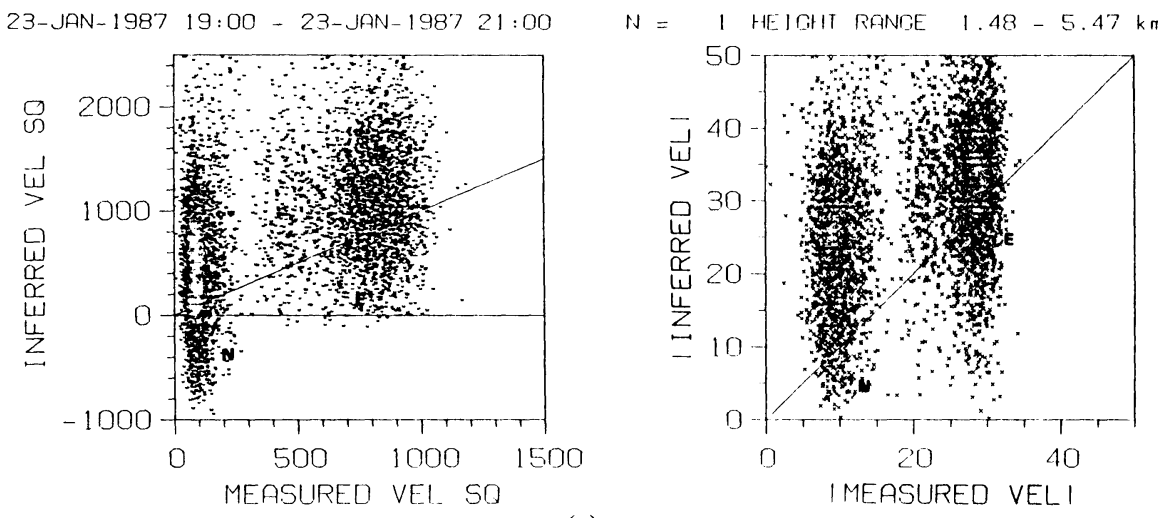

(a)
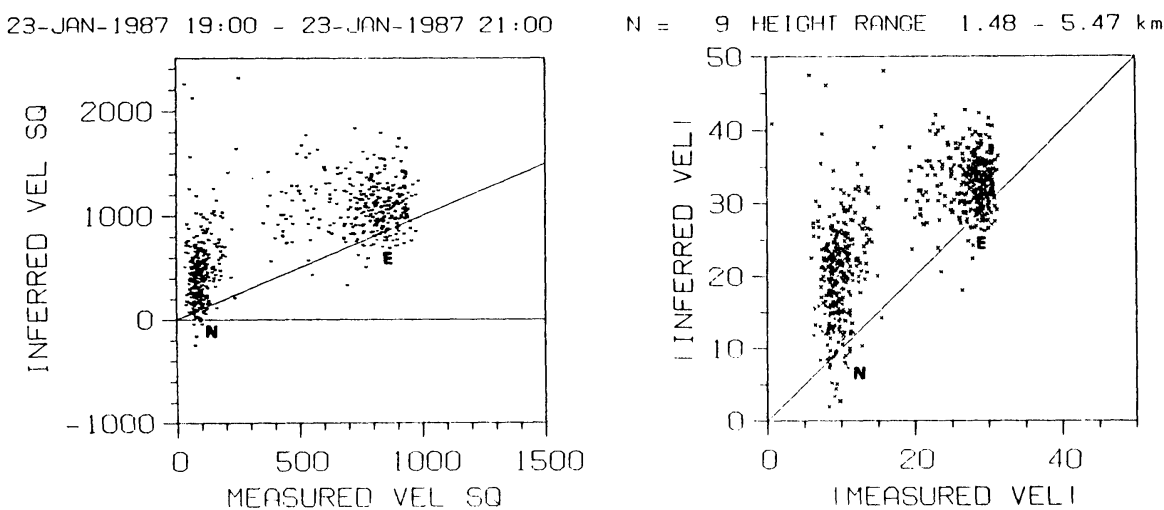

(b)
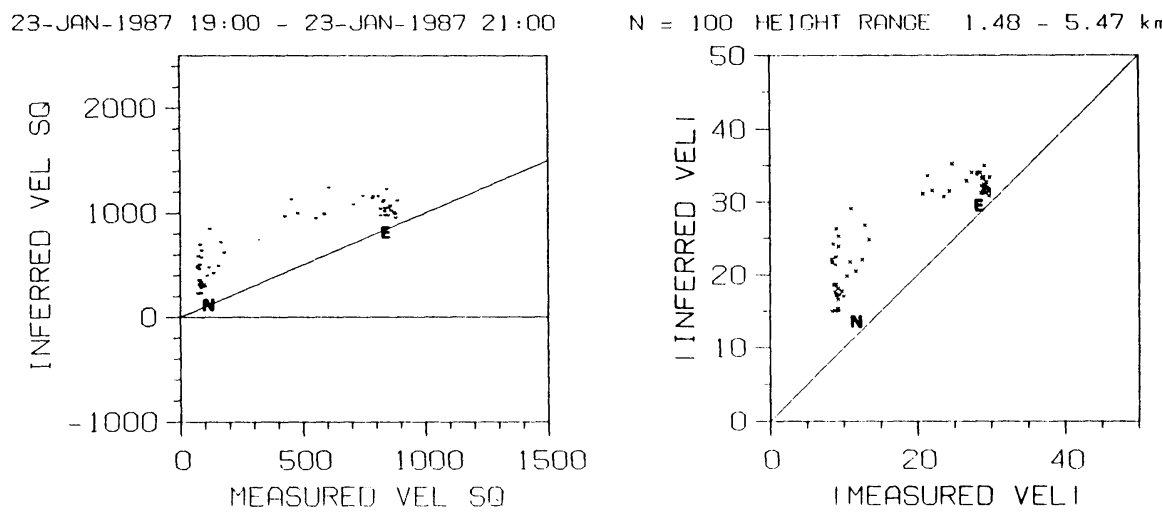

(c)

Fig. 2. Scatter plots of the magnitude of the velocity perpendicular to the radar beam inferred from the spectral width and mean Doppler shift against the direct estimate of the wind from the perpendicular beam. The plots are for cases where there were no post analysis averaging of the spectral width and Doppler shifts (a), and for cases where the spectral width was averaged over 9 (b) and 100 (c) samples. There are two distinct groups visible, with the smaller velocity associated with the estimates of the northward wind component and the larger being estimates of the eastward wind component and which are marked as $\mathrm{N}$ and $\mathrm{E}$ respectively. 


\section{Discussion and Conclusions}

An application of this technique may be useful for widely separated wind measurements, but for MST (or wind profiling) radars the spatial separation of the radar beams to estimate the horizontal wind vector is small, so that errors due to the spatial variability of the wind are small (e.g., STRAUCH et al., 1987). The wind measurements with Doppler weather radars are often widely separated but as was noted before, in these radars turbulence and windshear often have the largest contributions to spectral width. Great caution must be exercised as it was seen that the presence of even small windshear and turbulence may introduce substantial errors in the wind estimate. In many cases, such as during thunderstorms, by far the dominant contribution to spectral width is due to turbulence (e.g., DovIAK and ZRNIC, 1984, and references cited within). The precision of the estimate (as distinct from the error) of the wind component normal to the radar beam is similar to that obtained from measurements close together in azimuth (beams separated by about $\left.5-10^{\circ}\right)$.

This paper presents a method of obtaining estimates of the magnitude of the wind speed normal to the radar beam. These estimates represent a crude upper bound for this component of the wind. These results show reasonable consistency with the observed winds normal to a radar beam and may have some limited applications in Doppler weather radar studies. However, it must be stressed that even moderate broadening of the observed spectra due to windshear and turbulence has severe effects on the accuracy of the wind estimates.

One of us (PTM) was supported by an Australian Academy of Science/Japan Society for the Promotion of Science international exchange fellowship. The MU radar belongs to and is operated by the Radio Atmospheric Science Center of Kyoto University.

\section{REFERENCES}

Atlas, D., Advances in Geophysics, Vol. 10, 317 pp., Academic New York, 1964.

DoviaK, R. J. and D. S. ZRNIC, Doppler Radar and Weather Observations, 400 pp., Academic Press, Fla., 1984.

Fukao, S., T. Sato, T. Tsuda, S. Kato, K. Wakasugi, and T. Makihira, The MU radar with an active phased array system 1. Antenna and power amplifiers, Radio Sci., 20, 1155-1168, 1985a.

Fukao, S., T. Tsuda, T. Sato, S. Kato, K. Wakasugi, and T. Makihira, The MU radar with an active phased array system 2. In-house equipment, Radio Sci., 20, 1169-1176, 1985 b.

HockING, W. K., Measurement of turbulent energy dissipation rates by radar techniques: A review, Radio Sci., 20, 1403-1422, 1985.

HockING, W. K., Observation and measurement of turbulence in the middle atmosphere with a VHF radar, J. Atmos. Terr. Phys., 48, 655-670, 1986.

Strauch, R. G., W. L. Weber, A. S. Frisch, C. G. Little, D. A. Merritt, K. P. Moran, and D. C. WELSH, The relative accuracy of profiler wind measurements, J. Atmos. Ocean Tech., 4, 563-571, 1987.

Yamamoto, M., T. Sato, P. T. May, T. Tsuda, S. Fukao, and S. Kato, Estimation error of spectral parameters of MST radars obtained by a least square fitting method, submitted to Radio Sci., 1988. 